ISSN: 2320-7167

Volume 09 Issue 10 October 2021, Page no. - 2439-2442

Index Copernicus ICV: 57.55, Impact Factor: 7.184

DOI: $10.47191 / \mathrm{ijmcr} / \mathrm{v} 9 \mathrm{i10.08}$

\title{
Modelling of Nonlinear Problem Masstransfer in Three-Layer System
}

\section{Rustam Karjavovich Berdiev}

Senior Lecturer, Department of Informatics and Management National Institute of fine art and Design named after Kamoliddin Behzod Tashkent, Uzbekistan

\begin{tabular}{ll}
\hline ARTICLE INFO & ABSTRACT \\
\hline Published Online: & This article discusses modeling of nonlinear system of the differential equations in private \\
28 October 2021 & derivatives of parabolic type. Especially, modelling of nonlinear problem masstransfer in three-layer \\
Corresponding Author: & system will be analyzed in details. \\
Rustam Karjavovich & \\
Berdiev & \\
\hline KEYWORDS: Modeling Of Nonlinear System, Parabolic Type, Masstransfer \\
\hline
\end{tabular}

\section{INTRODUCTION}

We proceed from the following nonlinear system of differential equations

$$
\left.\begin{array}{l}
\varepsilon(t)-w_{0}\left(1-\frac{m_{s}-y}{m_{s}-y_{k p}}\right)+\frac{k_{1}}{2} \frac{\partial^{2} y^{2}}{\partial x^{2}}=\mu_{1} \frac{\partial y}{\partial t}+k_{2} \frac{y-h}{m_{2}} \\
\frac{T}{a} \frac{\partial h}{\partial t}=T \frac{\partial^{2} h}{\partial x^{2}}+T \chi(H-h)+\frac{k_{2}}{m_{2}}(y-h)
\end{array}\right\},
$$

Where $w_{0}, m_{s}, y_{k p}, k_{1}, k_{2}, m_{2}, \mu_{1}, a, T, \chi, H$ - some positive constants; $\varepsilon(t)$ - continuous differentiable function ; $t$; $y(x, t), h(x, t)$ - the desired functions. Introducing notation

$$
a_{1}=\frac{w_{0} y_{k p}}{m_{B}-y_{k p}} ; \quad b_{1}=\frac{a_{1}}{y_{k p}} ; \quad \mu_{2}=\frac{k_{2}}{m_{2}} ; \quad \mu_{3}=\frac{T}{a} ;
$$

The main results and findings

We will rewrite the system (1) in the form

$$
\left.\begin{array}{l}
\mu_{1} \frac{\partial y}{\partial t}=\frac{k_{1}}{2} \frac{\partial^{2} y^{2}}{\partial x^{2}}-\mu_{2}(y-h)+a_{1}-b_{1} y+\varepsilon(t) \\
\mu_{3} \frac{\partial h}{\partial t}=T \frac{\partial^{2} h}{\partial x^{2}}+T \chi(H-h)+\mu_{2}(y-h)
\end{array}\right\}
$$

are looking for the solution of system (2) in the form

$$
\left.\begin{array}{l}
y(x, t)=\alpha(t)(L-x)^{2}+\beta(t) \\
h(x, t)=\varphi(t)(L-x)^{2}+\psi(t)
\end{array}\right\},
$$

Where $\alpha(t), \beta(t), \varphi(t)$ и $\psi(t)$ - doubly differentiable functions to be defined. In this case, system (3) automatically satisfies the conditions 


$$
\left.\frac{\partial y}{\partial x}\right|_{x=L}=\left.\frac{\partial h}{\partial x}\right|_{x=L}=0
$$

From (3) we find

$$
\begin{aligned}
& y^{2}(x, t)=\alpha^{2}(t)(L-x)^{4}+2 \alpha(t) \beta(t)(l-x)^{2}+\beta^{2}(t) ; \\
& \frac{\partial^{2} y^{2}}{\partial x^{2}}=12 \alpha^{2}(t)(L-x)^{2}+4 \alpha(t) \beta(t)
\end{aligned}
$$

Substituting (3) into (2) taking into account (4) we get

$$
\begin{aligned}
& \mu_{1}\left[\dot{\alpha}(L-x)^{2}+\dot{\beta}\right]=6 k_{1} \alpha^{2}(t)(L-x)^{2}+2 k_{1} \alpha(t) \beta(t)+a_{1}-b_{1}\left[\alpha(t)(L-x)^{2}+\beta(t)\right]+ \\
& +\varepsilon(t)-\mu_{2}[\alpha(t)-\varphi(t)](L-x)^{2}-\mu_{2}[\beta(t)-\psi(t)] ; \\
& \mu_{3}\left[\dot{\varphi}(L-x)^{2}+\dot{\psi}\right]=2 T \varphi(t)+T \chi H-T \chi \varphi(t)(L-x)^{2}-T \chi \psi(t)+ \\
& +\mu_{2}[\alpha(t)-\varphi(t)](L-x)^{2}+\mu_{2}[\beta(t)-\psi(t)] .
\end{aligned}
$$

Equating the coefficients at $(L-x)^{2}$ and coefficients independent of $(L-x)^{2}$, we obtain the following nonlinear system of ordinary differential equations

$$
\begin{aligned}
& \mu_{1} \dot{\alpha}=6 k_{1} \alpha^{2}(t)-b_{1} \alpha(t)-\mu_{2}[\alpha(t)-\varphi(t)] ; \\
& \mu_{1} \dot{\beta}=2 k_{1} \alpha(t) \beta(t)+a_{1}-b_{1} \beta(t)+\varepsilon(t)-\mu_{2}[\beta(t)-\psi(t)] ; \\
& \mu_{3} \dot{\varphi}=-T \chi \varphi(t)+\mu_{2}[\alpha(t)-\varphi(t)] ; \\
& \mu_{3} \dot{\psi}=2 T \varphi(t)+T \chi H-T \chi \psi(t)+\mu_{2}[\beta(t)-\psi(t)] .
\end{aligned}
$$

From equation (5) we find

$$
\varphi(t)=\frac{1}{\mu_{2}}\left(\mu_{1} \dot{\alpha}+b_{1} \alpha-6 k_{1} \alpha^{2}\right)+\alpha=\frac{1}{\mu_{2}}\left(\mu_{1} \dot{\alpha}+6 k_{1} \alpha^{2}\right)+\frac{b_{1}+\mu_{2}}{\mu_{2}} \alpha
$$

From where follows

$$
\dot{\varphi}(t)=\frac{1}{\mu_{2}}\left[\mu_{1} \ddot{\alpha}-12 k_{1} \alpha \dot{\alpha}+\left(b_{1}+\mu_{2}\right) \dot{\alpha}\right]
$$

Relations (9) and (10) are substituted in (7)

$$
\begin{aligned}
& \frac{\mu_{3}}{\mu_{2}}\left[\mu_{1} \ddot{\alpha}-12 k_{1} \dot{\alpha} \dot{\alpha}+\left(b_{1}+\mu_{2}\right) \dot{\alpha}\right]=-\frac{T \chi}{\mu_{2}}\left[\mu_{1} \dot{\alpha}-6 k_{1} \alpha^{2}+\left(b_{1}+\mu_{2}\right) \alpha\right]- \\
& -\mu_{1} \dot{\alpha}-b_{1} \alpha+6 k_{1} \alpha^{2} .
\end{aligned}
$$

After the reduction of such, we come to the equation

$$
\begin{aligned}
& \frac{\mu_{1} \mu_{3}}{\mu_{2}} \ddot{\alpha}-\frac{12 k_{1} \mu_{3}}{\mu_{2}} \dot{\alpha} \dot{\alpha}+\frac{1}{\mu_{2}}\left[\mu_{3}\left(b_{1}+\mu_{2}\right)+\mu_{1} T \chi+\mu_{1} \mu_{2}\right] \dot{\alpha}- \\
& -\frac{6 k_{1}}{\mu_{2}}\left(\mu_{2}+T \chi\right) \alpha^{2}+\frac{1}{\mu_{2}}\left[b_{1} \mu_{2}+\left(b_{1}+\mu_{2}\right) T \chi\right] \alpha=0 \text {, or abbreviating to } \mu_{2} \text {, we get } \\
& \mu_{1} \mu_{3} \ddot{\alpha}-12 k_{1} \mu_{3} \alpha \dot{\alpha}+\left[\mu_{3}\left(b_{1}+\mu_{2}\right)+\mu_{1} T \chi+\mu_{1} \mu_{2}\right] \dot{\alpha}-6 k_{1}\left(\mu_{2}+T \chi\right) \alpha^{2}+
\end{aligned}
$$




$$
+\left[b_{1} \mu_{2}+\left(b_{1}+\mu_{2}\right) T \chi\right] \alpha=0
$$

Regarding the function $h(x, t)$ let's introduce an additional condition

$\left.\frac{\partial h}{\partial x}\right|_{x=0}=\varpi(t)$

where $\varpi(t)$ - a known doubly differentiable function of $t$, then it follows from the second equation of system (3) that

$$
\varphi(t)=-\frac{1}{L} \varpi(t)
$$

As a result, the general Riccati equation follows from (9)

$$
\dot{\alpha}=A_{1} \alpha^{2}-A_{2} \alpha+\lambda(t)
$$

By staging $\alpha=E(t) u(t)$, где $E(t)=\exp \int\left(-A_{2}\right) d t$

leads equation (14) to the form

$$
\dot{u}=A_{1} e^{-A_{2} t} u^{2}+\lambda(t) e^{A_{2} t}, \text { т.е. }
$$

the linear term vanishes. By asking $\varpi(t)$, and therefore and $\lambda(t)$ decision $u(t)$ you can write out [1] and write on the basis of (11) the following equation

$$
\ddot{\alpha}-12 \frac{k_{1}}{\mu_{1}} \alpha \dot{\alpha}+B_{1} \dot{\alpha}-6 \frac{k_{1}}{\mu_{1} \mu_{3}} \alpha^{2}=A_{3} \zeta(t),
$$

which, when substituting solution (14), turns into an identity.

We are interested in an independent solution with a possible change in the right part (16)

Conclusion

In [1] the method of obtaining the solution of equation f. 6.37 is specified (p.490)

$y^{\prime \prime}+2 y y^{\prime}+f(x)\left(y^{\prime}+y^{2}\right)=g(x)$ using substitution

$v(x)=y^{\prime}+y^{2}$, which boils down to $v^{\prime}+f(x) v=g(x)$.

In our case, it is necessary to use the substitution

$w(t)=\alpha^{\prime}-\frac{6 k_{1}}{\mu_{1} \mu_{3} B}$ and counting the variations of the left honor constant (16) of consciousness alignment to (17).

Functions $\beta(t)$ and $\psi(t)$ are found by integrating (6) and (8).

\section{REFERENCES}

1. Kamke E. Handbook of Ordinary differential equations M.: Nauka, 1971.-from 576.

2. Tikhonov A.N., Samarskiy A.A. Equations of mathematical physics. M.: Nauka. - 1977, 735c.

3. Polubarinova-Kochina P.Ya. Theory of groundwater movement.Moscow: Nauka. - 1977, 664s.

4. Barenblatt G.I., Kolesnikov V.M., Ryzhik V.M. Theory of unsteady filtration of liquid and gas. M.: Nedra. - 1972, 288s.

5. Stepanov V.V. Course of differential equations. M.: State Publishing House of Physics and Mathematics. literatures.1959, 468s.

6. Fichtenholz G.M. Course of differential and integral calculus, vol. II, M.: Nauka. - 1969, 800s.

7. Dwight G.B. Tables of integrals and other mathematical formulas. M.: Nauka. - 1978, 224c.

8. Ravshanov N., Kodirov K. Modeling of the process of filtration of groundwater in a well-permeable layer and its protection from pollution sources. - Voronezh, 2017. - Issue 3 (105), pp.170-181.

9. Information management as a field of scientific and practical activity. International journal on orange technologies www.journalsresearchparks.org/index.php/IJOT e- ISSN: Volume: 03 Issue: 02 | February 2021

10. Animation graphics in the presentation. International journal on human computing studies www.journalsresearchparks.org/index.php/IJHCS e-ISSN: 2615-8159|p-ISSN: 2615-1898 Volume: 03 Issue: 1 JanuaryFebruary 2021 
11. Sayfullaev, N. B. (2021). The Main Trends in the Development of Theatrical Art in Uzbekistan. International Journal of Early Childhood Special Education, 13(1).

12. Kurbanova, D. A. (2016). ANCIENT PEARLS IN GREAT WORLD MUSEUMS WHICH BELONG TO THE HISTORY OF UZBEKISTAN AND LEARNING THEM. Социосфера, (3), 55-61.

13. Kurbanova, D. (2020). Tent Of The Emir Of Bukhara (Second Half Of The 19th Century)(History Of One Exhibit From The Collection Of The State Hermitage). The American Journal of Social Science and Education Innovations, 2(12), $180-183$. 